\title{
A method for relaxing the Courant-Friedrich-Levy condition in time-explicit schemes
}

\author{
A. Hujeirat \\ Institute of Applied Mathematics, University of Heidelberg, 69120 Heidelberg, Germany \\ e-mail: ahmad.hujeirat@iwr.uni-heidelberg.de \\ Received 25 June 2004 / Accepted 1 October 2004 \begin{abstract}
explicit numerical methods in computational fluid dynamics. The method is based on re-formulating explicit methods in matrix form. Here an explicit method appears as a special case in which the global matrix of coefficients is replaced by the most simple matrix in algebra: the identity matrix I. This procedure is stable under severe limiting conditions only.

Using matrix formulation, one can design various solution methods in arbitrary dimensions that range from explicit to unconditionally stable implicit methods in which the CFL-number may reach arbitrary large values.

In addition, we find that adopting a specially varying-time-stepping scheme accelerates convergence toward steady state solutions and improves the efficiency of the solution procedure.
\end{abstract} \\ Abstract. We present a method for relaxing the Courant-Friedrich-Levy (CFL) condition, which limits the time step size in
}

Key words. methods: numerical - hydrodynamics - magnetohydrodynamics (MHD) - radiative transfer

\section{Introduction}

The majority of the numerical approaches used in astrophysical fluid dynamics (AFD) is based on time-explicit methods (Stone \& Norman 1992; Ziegler 1998; Koide et al. 1999).

These methods rely on time-extrapolation procedures for advancing the solution in time. However, in order to provide physically consistent solutions it is necessary that these procedures are numerically stable. The usual approach to examining the stability of numerical methods is to perform the so-called von Neumann analysis (see Hirsch 1988 for further details). For example, using an explicit procedure to solve the simple advection-diffusion equation:

$\frac{\partial T}{\partial t}+u \frac{\partial T}{\partial x}=v \frac{\partial^{2} T}{\partial x^{2}}+f$,

and applying the von Neumann stability analysis, it is necessary, but not sufficient, that the Courant-Friedrich-Levy (CFL) number is less than unity. This is equivalent to requiring that the time step size fulfills the inequality:

$\delta t \leq \min \left(\frac{\Delta X}{|u|+\left|\frac{\partial f}{\partial \rho}\right|}, \frac{\Delta X^{2}}{v}\right)$,

where $T, u, v, f, \rho$ denote an arbitrary diffusible variable, advective velocity, viscosity coefficient, internal or external forces and density of matter, respectively. The minimum function in the last inequality runs over each grid point of the domain of calculation. For additional details see Sect. 9.4 in Fletcher (1988). The force f may correspond to internal forces, such as thermal, radiative, or magnetic pressure. In regions where the density is low (e.g., the corona around compact objects such as black holes or even above accretion disks), the derivative $\left|\frac{\partial f}{\partial \rho}\right|$ corresponds to the sound speed, speed of light or of magnetic Alfvén waves, all of which can be extremely large compared to the actual velocity of the flow. Consequently, the CFL-condition limits the range of applicability and severely affects their robustness (see Fig. 1). In particular, equations corresponding to physical processes occurring on much shorter time scales than the hydro-time scale (e.g., radiation, self-gravitation and chemical reactions) cannot be followed explicitly. Furthermore, these methods are not suited for searching solutions that correspond to evolutionary phases occurring on time scales much longer than the hydro-time scale. Using high performance computers to perform a large number of explicit time steps may lead to an accumulation of roundoff errors that can easily distort the propagation of information from the boundaries and cause divergence of the solution procedure, especially if Neumann-type conditions are imposed at the boundaries.

In this paper we present for the first time a numerical strategy toward relaxing the CFL-condition, and therefore enlarging the range of application of explicit methods.

\section{Mathematical formulation - scalar case}

In fluid flows, the equation describing the time-evolution of a conservative quantity $q$ reads:

$\frac{\partial q}{\partial t}+L q \boldsymbol{V}=f$ 


\section{-...-Implicit ][ Explicit ][ Implicit -..- \\ $\tau<\tau_{\mathrm{G}, \mathrm{R}}<<\tau_{\mathrm{Ch}}<\tau_{\mathrm{MF}}<\tau_{\mathrm{HD}}<\tau_{\mathrm{Th}}<<\tau_{\mathrm{vis}}<<\tau_{\mathrm{Ev}}$}

Fig. 1. The range of applicability of implicit and explicit methods. The equations describing physical and chemical processes whose characteristic time scales are much shorter than the hydrodynamic time scale $\left(\tau_{\mathrm{HD}}\right)$ should be solved implicitly. For example, the characteristic time corresponding to the propagation of gravitational waves $\left(\tau_{\mathrm{G}}\right)$, peaks in the radiative energy $\left(\tau_{\mathrm{R}}\right)$ and chemical processes $\left(\tau_{\mathrm{Ch}}\right)$ occur on much shorter time scales than $\tau_{\mathrm{HD}}$. In most astrophysical problems the Alfvén wave crossing time $\left(\tau_{\mathrm{MF}}\right)$ is shorter than $\tau_{\mathrm{HD}}$. The time scale required for fluid flows (non-relativistic) to relax thermally $\left(\tau_{\mathrm{Th}}\right)$ is in general one order of magnitude larger than $\tau_{\mathrm{HD}}$, depending on the efficiency of the cooling processes. However, the viscous time scale $\left(\tau_{\mathrm{Vis}}\right)$ can be significantly larger than $\tau_{\mathrm{HD}}$ depending on how large the Reynolds number $(\mathrm{Re})$ is. In most astrophysical flows, $\mathrm{Re}$ is at least $10^{3}$. If accretion is considered, the time scale for an envelope to evolve $\left(\tau_{\mathrm{Ev}}\right)$ is approximately equal to the mass of the envelope divided by the accretion rate. Thus, for an envelope of $\mathcal{M}_{\mathrm{En}}=10^{-5} M_{\odot}$ to evolve from an accretion rate of $\dot{\mathcal{M}} \sim 10^{-10} M_{\odot} / Y$, an accretion time of the order $\tau_{\mathrm{Ev}} \sim 10^{5}$ years is required.

where $\boldsymbol{V}$ and $f$ are spatially varying velocity-field and external forces, respectively. $L$ represents a first and/or second order linear differential operator that describe the advection and diffusion of $q$.

In the finite space $\mathcal{R}$ we may replace the time derivative of $q$ by:

$\frac{\delta q}{\delta t}=\frac{q^{n+1}-q^{n}}{\delta t}$

where $q^{n}$ and $q^{n+1}$ correspond to the actual value of $q$ at the old and new time levels, respectively.

An explicit formulation of Eq. (3) reads:

$\frac{\delta q}{\delta t}=[-L q \boldsymbol{V}+f]^{n}$

whereas the corresponding implicit form is:

$\frac{\delta q}{\delta t}=[-L q \boldsymbol{V}+f]^{n+1}$.

Combining these two approaches, we obtain:

$R=\frac{\delta q}{\delta t}-\theta[-L q \boldsymbol{V}+f]^{n+1}-(1-\theta)[-L q \boldsymbol{V}+f]^{n}$

where $R$ is the residual, and $\theta(0 \leq \theta \leq 1)$ is a switch on/off parameter.

Applying a conservative discretization, we may proceed to calculate the elements of the Jacobian on the new time level:

$J_{j, k}=\left[\frac{\partial R_{j}}{\partial q_{k}}\right]^{n+1}$.

The Jacobian $J$ can be decomposed as follows:

$J=\frac{I}{\delta t}+\theta A$

where

$A=\frac{\partial}{\partial q}[-L q \boldsymbol{V}+f]^{n+1}$.

Thus, the matrix equation that corresponds to Eq. (7) reads:

$\left(\frac{I}{\delta t}+\theta A\right) \delta q=\operatorname{RHS}^{n}$

The matrix $A$ contains coefficients such as $V / \Delta x, \eta / \Delta x^{2}$ that correspond to advection and diffusion terms, respectively, as well as additional coefficients that correspond to source terms.
In terms of Eq. (8), explicit methods are recovered by neglecting the matrix $\theta A$. We note that since the switch parameter $\theta$ does not appear on the RHS of Eq. (8), explicit formulation does not depend directly on $\theta$, but rather on the multiplication of $\theta A$. Thus, for an explicit method to converge it is necessary that the matrix $\left(\frac{I}{\delta t}+\theta A\right)$ is diagonally dominant, but also that $\theta A$ is negligibly small compared to $I / \delta t$. In terms of matrix algebra, this means that the absolute value of the sum of the elements in each row of $\theta A$ must be smaller than the corresponding diagonal element $1 / \delta t$ (Hackbusch 1994). In the absence of diffusion and external forces (i.e., $\eta=f=0$ ), this is equivalent to the requirement that at each grid point the following inequality must hold:

$|\Delta V q| / \Delta x<|q| / \delta t$

where $q$ may acquire negative and positive values. However, since $V q$ is a conservative quantity, we may obtain an upper limit for the flux difference:

$|\Delta V q| \leq \max \left\{(V q)_{\text {in }},(V q)_{\text {out }}\right\}=|V||q|$,

where the subscripts "in, out denote the locations of the in- and out-flows through the surfaces of an arbitrary finite volume cell. In writing the last equality, we have omitted these subscripts for simplicity.

Thus, in terms of Eq. (8), neglecting $\theta A$ is equivalent to requiring:

$|\Delta V q| / \Delta x \leq|V||q| / \Delta x<|q| / \delta t$.

This is equivalent to the outcome of the von Neumann stability analysis (see Richtmyer \& Morton 1967), which yielded the well-known condition $C F L=|V| \delta t / \Delta x<1$.

It should be noted, however, that the classical derivation of the CFL conditions relies on the assumption that the velocity $V$ in Eq. (1) is constant. Practically, the HD-equations are nonlinear, and using $C F L=1$ does not prevent the exponential growth of the instability. Indeed, most of the explicit methods used in CFA provide stable solutions, if only the CFL-number is strictly less than unity, and in most cases, a $C F L \leq 0.6$ is required.

The matrix $A$ can be decomposed as follows: $A=D+L+$ $U$, where $D$ is a matrix that consists of the diagonal elements of $A$. $L$ and $U$ contain respectively the sub- and super-diagonal entries of $A$. Noting that a conservative discretization of the 
advection-diffusion hydrodynamical equations (Navier-Stokes equations) gives rise to a $D$ which contains positive values, we may reconstruct a modified diagonal matrix $D_{\text {mod }}=I / \delta t+\theta D$. In this case, Eq. (8) is equivalent to:

$\left[D_{\text {mod }}+\theta(L+U)\right] \delta q=\mathrm{RHS}^{n}$.

A slightly modified semi-explicit form can be obtained by neglecting the entries of the matrix $\theta(L+U)$. In this case, a necessary condition for the iteration procedure to converge is that the absolute value of the sum of the elements in each row of $\theta(L+U)$ must be much smaller than the corresponding diagonal element of $D_{\text {mod }}$ in the same row. In terms of Eq. (9), the method is said to converge if the entries in each row of $D_{\text {mod }}$ fulfill the following condition:

$1 / \delta t+\theta\left(|V| / \Delta x+\eta / \Delta x^{2}+g\right)>\|A-D\|_{\infty}$,

where $\|A-D\|_{\infty}$ denotes the $\infty$-norm of $A-D$, i.e., the maximum row sum of the modulus of the elements of $A-D$. This condition can be fulfilled, however, if the flow is smooth and viscous, and if appropriate boundary conditions are imposed ${ }^{1}$. Consequently, the inversion process of $D_{\bmod } \delta q=$ RHS $^{n}$ should proceed stably even for large CFL-numbers (see Fig. 5).

Note that using $\theta<1$ yields a certain average of the $q$-values on the new and old time levels. This averaging process is useful if the sought solutions are strongly time-dependent, such as propagations of shock waves, where using large time steps may cause divergence of the solution track, or even numerically destabilize the scheme. A possible way to capture both time-dependent and quasi stationary solutions is to relate $\theta$ to the time-step size, which in turns depends on the total residual. An example is the damped Crank-Nicolson scheme: $\theta=(1+\alpha \delta t) / 2 \leq 1$, where $\alpha$ is a constant of order unity (see Hujeirat \& Rannacher 2001, references therein). If stationary solutions are of interest, $\theta=1$ should be used.

\section{Generalization}

The set of 2D-hydrodynamical equations in conservative form and in Cartesian coordinates may be written in the following vector form:

$\frac{\partial \boldsymbol{q}}{\partial t}+L_{x, x x} \boldsymbol{F}+L_{y, y y} \boldsymbol{G}=\boldsymbol{f}$

where $F$ and $G$ are fluxes of $q$, and $L_{x, x x}, L_{y, y y}$ are first and second order transport operators that describe advection-diffusion of the vector variables $\boldsymbol{q}$ in $x$ and $y$ directions. $\boldsymbol{f}$ corresponds to the vector of source functions.

By analogy with Eq. (6), when linearizing Eq. (14) the following matrix form can be obtained:

$\left[\frac{I}{\delta t}+\theta\left(A L_{x, x x}+B L_{y, y y}-H\right)\right] \delta q=\mathrm{RHS}^{n}$,

where $A[=\partial F / \partial q], B[=\partial G / \partial q]$ and $H[=\partial \boldsymbol{f} / \partial q]$, are evaluated on the former (or the new, if the Newton method

\footnotetext{
${ }^{1}$ Note that diffusion makes the inequality in Eq. (13) more pronounced, giving rise to larger CFL-numbers.
}

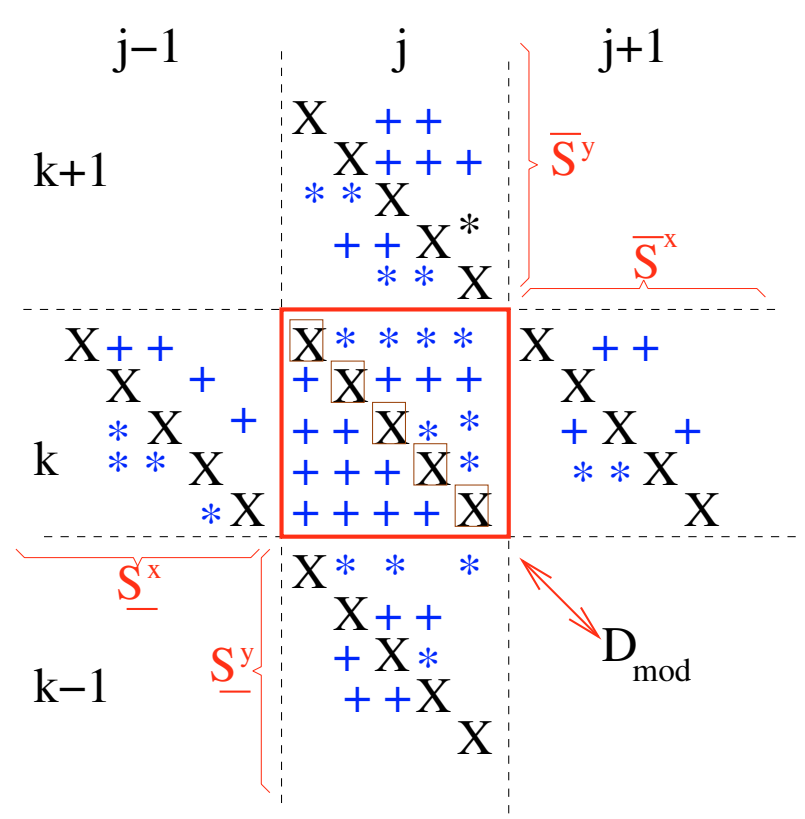

Fig. 2. Block matrices in the $x$ and $y$-directions resulting from 5-star staggered grid discretization. Entries marked with " $X$ " denote the elements usually used in the implicit operator-splitting approach (Hujeirat \& Rannacher 2001), "*” and "+" are coefficients corresponding to the source terms. The semi-explicit method for a scalar equation relies on inverting the diagonal matrix whose entries are marked with $X$ surrounded by squares. The generalization of the semiexplicit method to the multi-dimensional HD-equations requires inverting the block diagonal matrix $D_{\text {mod }}$.

is used to construct the matrices) time levels. $\mathrm{RHS}^{n}=[\boldsymbol{f}-$ $\left.L_{x, x x} \boldsymbol{F}-L_{y, y y} \boldsymbol{G}\right]^{n}$.

Adopting a five star staggered grid discretization, it is easy to verify that at each grid point Eq. (15) corresponds to the following block matrix equation:

$$
\begin{aligned}
& \frac{\delta q_{j, k}}{\delta t}+\underline{S}^{x} \delta q_{j-1, k}+D^{x} \delta q_{j, k}+\bar{S}^{x} \delta q_{j+1, k} \\
& +\underline{S}^{y} \delta q_{j, k-1}+D^{y} \delta q_{j, k}+\bar{S}^{y} \delta q_{j, k+1}=\mathrm{RHS}_{j, k}^{n},
\end{aligned}
$$

where the underlines (overlines) mark the sub-diagonal (superdiagonal) block matrices in the corresponding directions (see Fig. 2). $D^{x, y}$ are the diagonal block matrices resulting from the discretization of the operators $L_{x, x x} \boldsymbol{F}, L_{y, y y} \boldsymbol{G}$ and $\boldsymbol{f}$.

To outline the directional dependence of the block matrices, we re-write Eq. (16) in a more compact form:

$$
\begin{aligned}
& \bar{S}^{y} \delta q_{j, k+1} \\
+\underline{S}^{x} \delta q_{j-1, k} & +D_{\bmod } \delta q_{j, k}+\bar{S}^{x} \delta q_{j+1, k}=\operatorname{RHS}_{j, k}^{n} \\
& +\underline{S}^{y} \delta q_{j, k-1} .
\end{aligned}
$$

where $D_{\text {mod }}=\delta q_{j, k} / \delta t+D^{x}+D^{y}$. The subscripts " $j$ " and " $k$ " denote the grid-numbering in the $x$ and $y$ directions, respectively (see Fig. 2). Equation (17) gives rise to three different types of solution procedures:

1. Classical explicit methods are obviously very special cases that are recovered when the sub- and super-diagonal block matrices together with $D^{x}$ and $D^{y}$ are neglected. The only 
matrix to be retained here is $(1 / \delta t) \times($ the identity matrix $)$, i.e., the first term on the LHS of Eq. (16). This yields the vector equation:

$$
\frac{\delta q_{j, k}}{\delta t}=\operatorname{RHS}_{j, k}^{n} \text {. }
$$

2. Semi-explicit methods are recovered when only the suband super-diagonal block matrices are neglected, but the block diagonal matrices are retained. In this case the matrix equation reads:

$$
D_{\text {mod }} \delta q_{j, k}=\operatorname{RHS}_{j, k}^{n} \text {. }
$$

We note that inverting $D_{\text {mod }}$ is a straightforward procedure, which can be performed analytically or numerically.

3. A fully implicit solution procedure requires retaining all the block matrices on the LHS of Eq. (17). This yields a global matrix that is highly sparse. In this case, the "Approximate Factorization Method" (-AFM: Beam \& Warming 1978) and the "Line Gauss-Seidel Relaxation Method" (-LGS: MacCormack 1985) are considered to be efficient solvers for such a set of HD-equations in multi-dimensions.

To verify the consistency and robustness of the method, Taylor flows between two concentric and rotating spheres have been tested. This is an ideal test problem, as the flow here is viscous (second-order diffusive operators are included), has closed boundaries (without external perturbations) and the corresponding equations accept strict time-independent solutions.

Figure 3 shows the time-development of the CFL-number and the total residual for 5 different solution procedures for searching steady state configurations for Taylor flows between two concentric spheres. Using spherical geometry, the set of the 2D axi-symmetric Navier-Stokes equations are solved. The set consists of the three momentum equations, the continuity and the internal energy equations. The flow is assumed to be adiabatic.

As boundary conditions, the inner sphere is set to rotate with $\Omega=5$, whereas the outer sphere has $\Omega=0$. The density, temperature, radial and angular velocities are set to be symmetric along the equator and along the polar axis, except the angular velocity which assumes anti-symmetric conditions along the axis of rotation. For the horizontal velocity, anti-symmetric conditions are imposed both along the equator and along the axis of rotation. On the inner and outer radii of the spheres all velocity components are set to vanish. Initially, the flow between the two spheres has zero poloidal and toroidal velocities, so that the rotational energy is injected into the flow through viscous interaction with the inner boundary. In these test calculations, the viscosity coefficient $\eta=10^{-2}$ and the switch on/off parameter $\theta=1$ have been used.

In the explicit case, the equations are solved according to Eq. (18). For the semi-explicit procedure, we solve the HD-equations using the block matrix formulation as described in Eq. (19). The implicit operator-splitting approach is based on solving each of the HD-equations implicitly. Here, the LGS method has been used in the inversion procedure of each equation (Hujeirat \& Rannacher 2001). Unfortunately, while this method has been proven to be robust for modeling compressible flows with open boundaries, it fails to achieve large

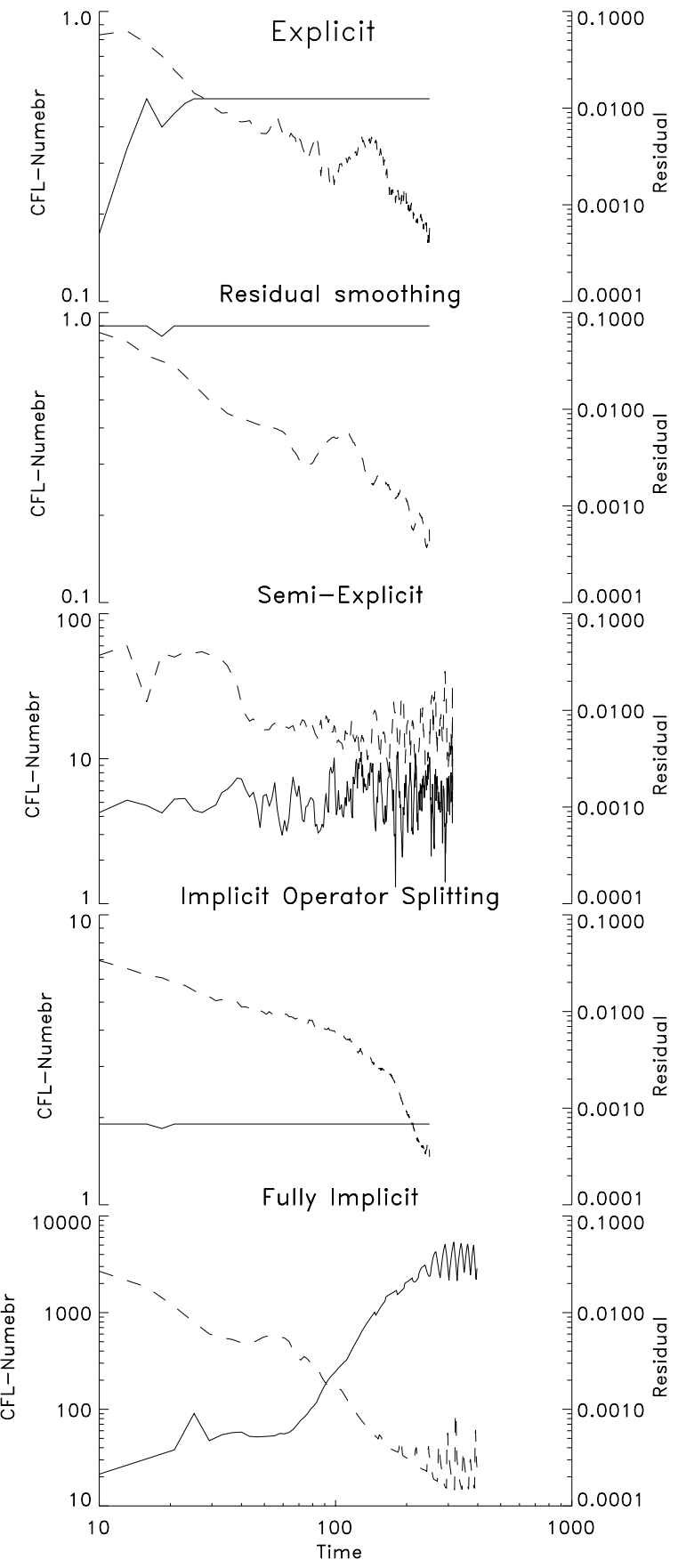

Fig. 3. The CFL number (left axis, solid line) and the total residual (right axis, dashed line) as a function of time in normalized units, using different numerical methods. The methods are (from top to bottom): normal explicit, residual smoothing, semi-explicit, implicit operator splitting (Hujeirat \& Rannacher 2001) and the fully implicit method. While the effective time reached in each run of these different methods is similar, the actual number of iterations is substantially different. The numerical problem here is to search stationary solutions for Taylor flow between two concentric spheres. The inner sphere has a radius $r_{\text {in }}=1$ and rotates with angular velocity $\Omega_{\text {in }}=5$, whereas the outer sphere is non-rotating and its radius is taken to be $r_{\text {out }}=1$.3. $\theta=1$ and $\eta=10^{-2}$ have been used in these calculations. The initial density and temperature are taken to be $\rho(r, \theta, t=0)=1$, and $T(r, \theta, t=0)=10^{1}$, respectively. The computational domain is $[1,1.3] \times[0, \pi / 2]$ and consists of $30 \times 50$ non-uniformly distributed tensor-product cells. 


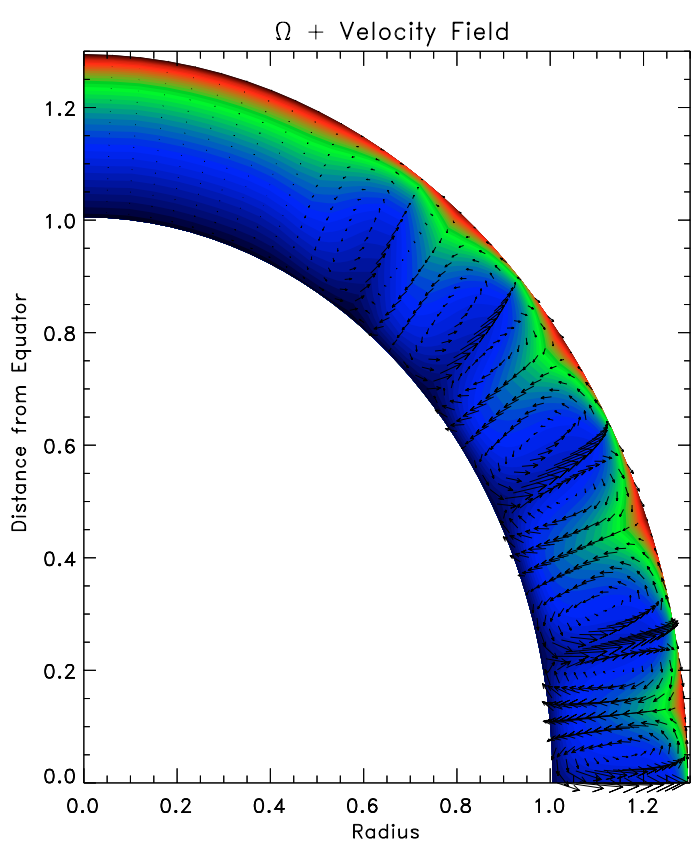

Fig. 4. Taylor flow between two concentric spheres. Here the velocity field and the angular velocity (large-to-low values correspond to blueto-red colors) are shown. The ability of the methods to capture the formation of rotationally-driven multiple vortices near the equatorial region is obvious.

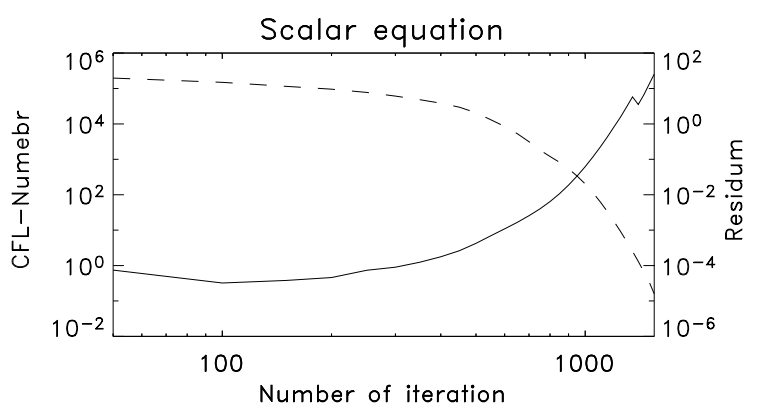

Fig. 5. Taylor flow between two concentric spheres. Here, we illustrate the semi-explicit method applied to the angular momentum equation. Similar to Fig. 3, this figure shows the development of the CFL-number (left axis, solid line) and the residual (right axis, dashed line) of the angular momentum equation in two dimensions versus the iteration number. As initial conditions we use the steady distributions of the physical variables that have been obtained from the simulations of the Taylor problem (see Fig. 4). This includes the velocity field, density, temperature, and $\eta$. For the angular velocity we use $\Omega=0$ as initial condition.

CFL-numbers in weakly incompressible flows (Fig. 3). This indicates that pressure gradients in weakly incompressible flows do not admit splitting, and therefore they should be included at the new time level.

Using the semi-explicit solution procedure, the CFL-numbers obtained in the present modelling of Taylor flows are, indeed, larger than unity (middle, Fig. 3), but they are not impressively large as we have predicted theoretically. We may attribute this inconsistency to three different effects: 1) The flow considered here is weakly incompressible. This means that the acoustic perturbations have the largest propagation speeds, which requires that all pressure effects should be simultaneously included in the solution procedure on the new time level. 2) The conditions imposed on the boundaries are non-absorbing, and do not permit advection of errors into regions exterior to the domain of calculation. 3) The method probably requires additional improvements in order to achieve large CFL-numbers. This could be done, for example, within the context of the "defect-correction" iteration procedure, in which the block diagonal matrix $D_{\text {mod }}$ is employed as a pre-conditioner.

In the final case, the whole set of HD-equations is solved taking into account all pressure terms in a fully implicit manner (Fig. 3/bottom). Here we use the AFM for solving the general block matrix equation which is locally described by Eq. (17).

For controlling the time step size in these calculations we have adopted the following description: $\delta t=\alpha_{0} \epsilon / \max \left(\operatorname{RHS}_{j, k}\right)$, where $\epsilon=\min \left(\Delta X_{j}, \Delta Y_{k}\right)$, and where $\alpha_{0}$ is a constant of order unity. The maximum and minimum functions here are set to run over the whole number of grid points.

Note the difference between the terminal values of the residual in the semi-explicit and the fully implicit cases. After a certain number of iterations, the residual in the former case does not decrease, but, instead, it shows strong, amplitudelimited variations as the number of iterations is increased. Although a CFL-number of order 10 is achieved in this test calculation, the origin and magnitudes of these variations are not completely clear. However, taking into account that these strong variations do not show up in the fully implicit case, we may conclude that their origin is connected to the incomplete inclusion of the source terms in the coefficient matrix.

In these calculations, although the effective time reached in each of these runs is similar, the actual number of iterations performed is substantially different. Specifically, since the CPU time scales linearly with the number of computing operations performed, hence with the total computational costs $(C C)$, the following correlation was found:

$$
C C_{\mathrm{SE}}<C C_{\mathrm{ERS}}<C C_{\text {Explicit }}<C C_{\mathrm{IOS}}<C C_{\mathrm{FI}},
$$

where the subscripts have the following meanings: $\mathrm{SE}=$ Semiexplicit, ERS = explicit residual smoothing, IOS = implicit operator splitting and FI = fully implicit.

\subsection{The specially varying time stepping scheme for accelerating convergence}

Let $[a, b]$ be the interval on which Eq. (3) is to be solved. We may divide $[a, b]$ into $N$ equally spaced finite volume cells: $\Delta x_{\mathrm{i}}=(b-a) / N, i=1, N$. To follow the time evolution of q using a classical explicit method, the time step size must fulfill the CFL-condition, which requires $\delta t$ to be smaller than the critical value: $\delta t_{\mathrm{c}}^{\mathrm{u}}$ If $[a, b]$ is divided into $N$ highly stretched finite volume cells, for example $\Delta x_{1}<\Delta x_{2} \ldots<\Delta x_{N}$, then the CFL-condition restricts the time step size to be even smaller than $\delta t_{\mathrm{c}}^{\mathrm{nu}}=\min _{i}\left\{\left(\Delta x_{i} /\left(V+V_{\mathrm{S}}\right)_{i}\right\}\right.$, which is much smaller than $\delta t_{\mathrm{c}}^{\mathrm{u}}$. Thus, applying a conditionally stable method to model flows while using a highly non-uniform distributed mesh has the disadvantage that the time evolution of the variables in the 
whole domain are artificially and severely affected by the flow behaviour on the smallest cell.

Moreover, time-advancing the variables may stagnate if the flow is strongly or nearly incompressible. In this case, $V_{\mathrm{S}} \gg$ $V$, which implies that the time step size allowed by the CFL-condition approaches zero.

However, we may still associate a time step size with each grid point, e.g., $\delta t_{i}^{\mathrm{nu}}=\Delta x_{i} /\left(V+V_{\mathrm{S}}\right)$, and follow the time evolution of variable $q$ in each cell independently. Interactions between variables enter the solution procedure through the evaluation of the spatial operators on the former time level. This method, which is occasionally called the "Residual Smoothing Method" proved to be efficient at providing quasi-stationary solutions within a reasonable number of iterations, when compared to normal explicit methods (Fig. 3, also see Swanson $\&$ Turkel 1997; Enander 1997). The main disadvantage of this method is its inability to provide physically meaningful time scales for features that possess quasi-stationary behaviour. Here we suggest to use the obtained quasi-stationary solutions as initial configuration and re-start the calculations using a uniform and physically relevant time step size.

\section{Additional test calculations}

In the following, we present the results of several test calculations aimed to examine the stability, robustness and the capability of the semi-explicit solver to treat strongly time-dependent or steady inviscid flows governed by strong shocks.

\subsection{Free-fall of matter onto a Schwarzschild black hole}

In the absence of other resistive forces, a non-rotating gas around a spinless black hole is gravitationally bound, and therefore should fall free onto the black hole. The density and velocity profiles of free falling matter in the radial direction and far from the event horizon obey the power laws $r^{-3 / 2}$ and $r^{-1 / 2}$, respectively. This physical problem is relevant for testing the capability of the numerical approach at capturing steady and oscillation-free solutions, even when a strongly stretched mesh distribution is used.

The equations to be solved in this problem are the continuity, the radial and horizontal momentum equations, and the internal energy equation. The flow is inviscid and adiabatic with the adiabatic index $\gamma=5 / 3$. The equations have been solved using a first order accurate advection scheme both in space and time. In carrying out these calculations, the following conditions have been taken into account.

- The central object is a solar-mass non-rotating black hole.

- The outer boundary is 100 times larger than the inner radius, i.e., $R_{\text {out }}=100 \times R_{\text {in }}$.

- Along the outer boundary, the density and temperature of the gas assume uniform distributions and flow across this boundary with the free-fall velocity. Symmetry boundary conditions along the equator, and asymmetry boundary conditions along the axis of rotation have been imposed.
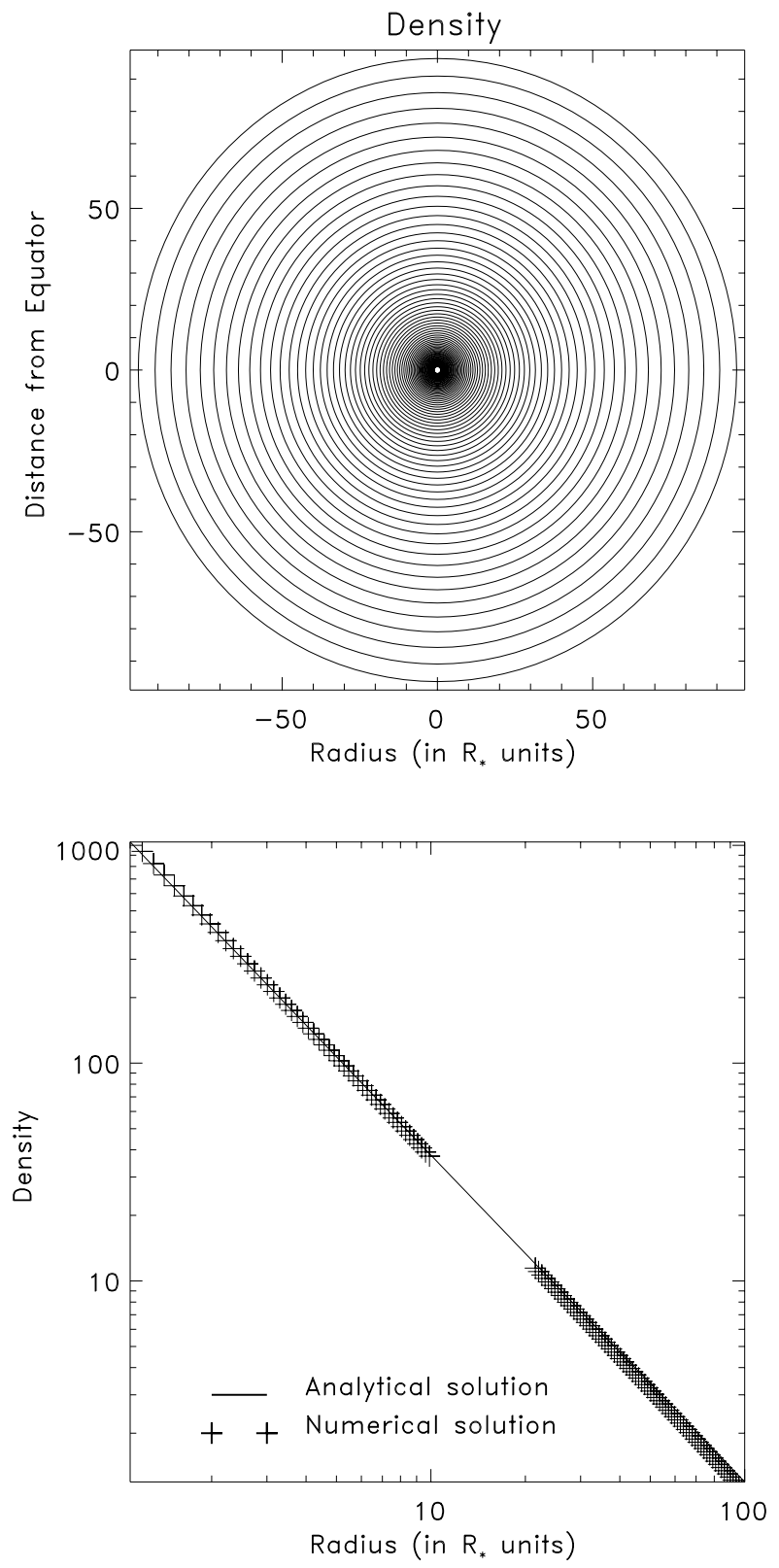

Fig. 6. 50 density contours of a freely-falling gas onto a one-solarmass and non-rotating black hole (left). Right: the numericallyobtained density distribution has a power law that coincides precisely with the theoretical solution $r^{-3 / 2}$. In this calculation, inflow (outflow) at the outer (inner) boundary has been imposed. 200 strongly-stretched finite volume cells in the radial direction and 60 in horizontal direction have been used.

Along the inner boundary, we have imposed non-reflecting and outflow conditions.

The above set of equations is solved in the first quadrant $[1 \leq$ $r \leq 100] \times[0 \leq \theta \leq \pi / 2]$, where 200 strongly stretched finite volume cells in the radial direction and 60 in the horizontal direction are used.

Figure 6 shows the 2D distribution of the density around the hole. The bottom plot of Fig. 6 shows the accurate agreement of the numerical with the theoretical solution. 

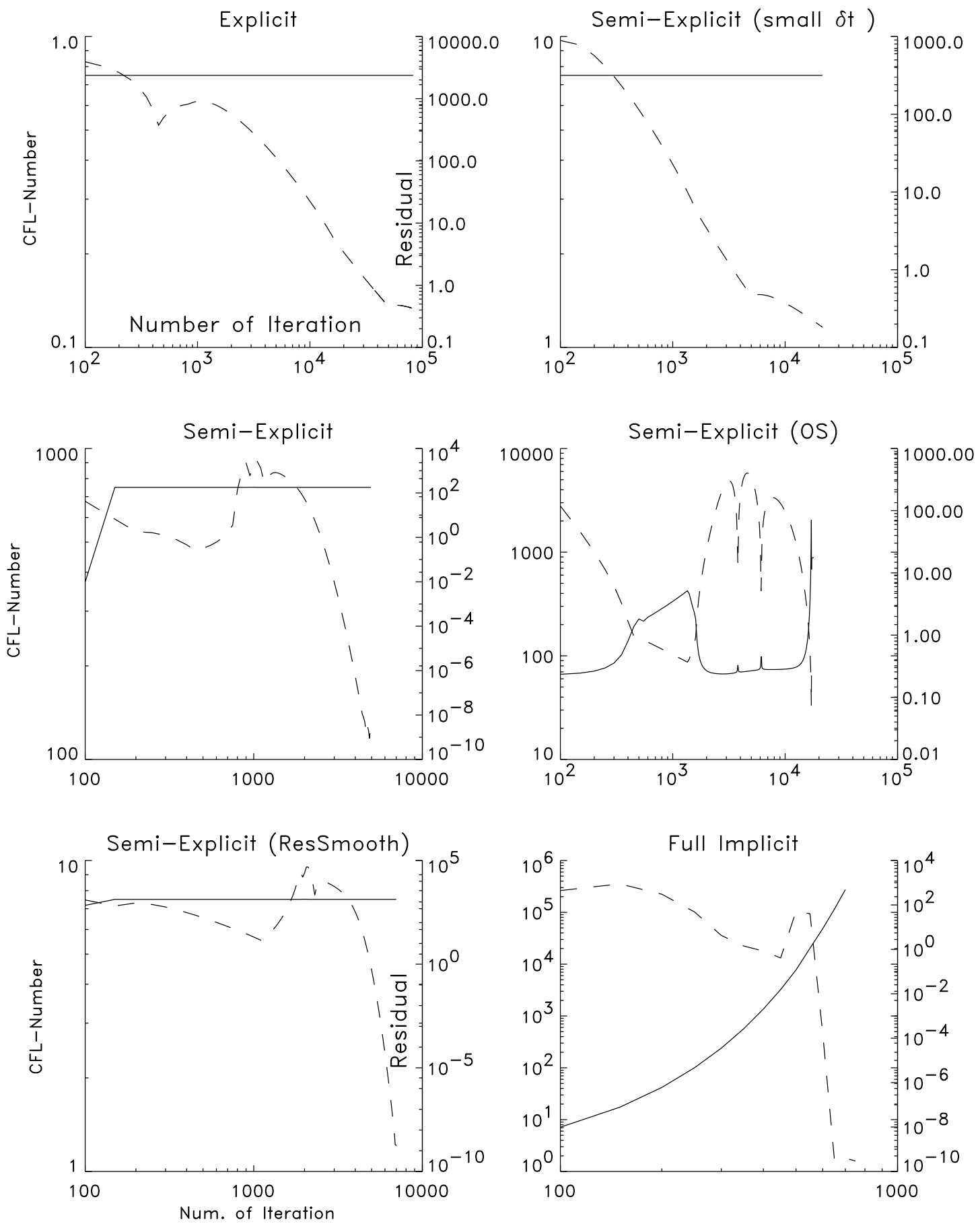

Fig. 7. Plasma in free-fall onto a black hole. Similar to Fig. 3, the evolution of the CFL-number and the residual versus iteration number are shown. The solution methods used here are: normal explicit (top/left), semi-explicit (middle/left), semi-explicit in combination with the residual smoothing strategy (bottom/left), semi-explicit using moderate CFL-numbers (top/right), semi-explicit method in which the time step size is taken to be a function of the maximum residual (middle/right), and finally the fully implicit method (bottom/right). Obviously, the different forms of the semi-explicit method used here are stable and converge to the stationary solution, though at different rates.

As in Fig. 3, we show in Fig. 7 the evolutions of the CFL-number and the residual as a function of the iteration number which has been obtained using different numerical approaches. The results show that the convergence of the explicit and of the semi-explicit methods is rather slow when a relatively small time step size is used. This implies that the amplitude-limited oscillations are strongly time-dependent, which may result from geometric compression. Indeed, these perturbations disappear when relatively large time steps are used (see Fig. 7, middle/right).

In addition, the semi-explicit solver has been tested in combination with the residual smoothing strategy. As expected, this approach accelerates convergence considerably (Fig. 7: compare the plots bottom/left and top/right). 
In most of the cases considered here, the time step is set to increase in a well-prescribed manner and independent of the residual. However, determining the size of the time step from the residual directly did not provide satisfactory convergence histories (Fig. 7, middle/right).

The results obtained here indicate that the semi-explicit method is stable compared to normal explicit methods. Furthermore, the semi-explicit method can be used to search for stationary solutions using large time steps, or equivalently, CFL-numbers that are significantly larger than unity (Fig. 7, middle/left).

\subsection{Free-fall onto a Schwarzschild black hole surrounded by a cold disk}

The test problem here is similar to the previous flow problem almost in every aspect, except that a cold and dense disk has been placed in the innermost equatorial region: $[1 \leq r \leq 10] \times$ $[-0.3 \leq \theta \leq 0.3]$. Conditions of vanishing in- and out-flow have been imposed at the boundaries of this disk. The plasma surrounding the disk is taken to be inviscid, thin, hot, and nonrotating. Thus, the flow configuration is similar to the forward facing step problem usually used for test calculations in CFD. The disk here serves as a barrier that prevents the gas from freely falling onto the black hole, and instead, it forms a curved shock front around the cold disk. The purpose of this test is mainly to examine the capability of the method at capturing steady solutions governed by strong shocks.

In solving the HD-equations (see Sect. 4.1), an advection scheme of third order spatial accuracy and first order accuracy in time has been used. The domain of calculation is subdivided into 200 strongly stretched finite volume cells in the radial direction and 60 in the horizontal direction. In Fig. 8 the steady distributions of the density, temperature and the velocity field are shown. Similar to the calculation in the previous sections, the results indicate that the semi-explicit method is stable and converges to the steady solution sought even when a CFL-number of order 200 is used (see Fig. 9). However, the method converges relatively slowly compared to the implicit operator-splitting approach, where steady solutions have been obtained after one thousand iterations only.

\subsection{Wave propagation}

In an attempt to examine the capability of the method at capturing strongly time-dependent features, the following onedimensional wave equation in spherical geometry has been solved:

$\frac{\partial \rho}{\partial t}+\frac{1}{r^{2}} \frac{\partial}{\partial r} r^{2} \rho U=0$

where $\rho, U$ denote the density and radial velocity, respectively. The velocity assumes the constant value $U=1$. To reduce the effect of geometrical compression, the interval of calculation is taken to be $[100 \leq r \leq 104]$. Similar to the previous test problems, different solution procedures have been adopted using advection schemes of third order spatial accuracy and second order accuracy in time.
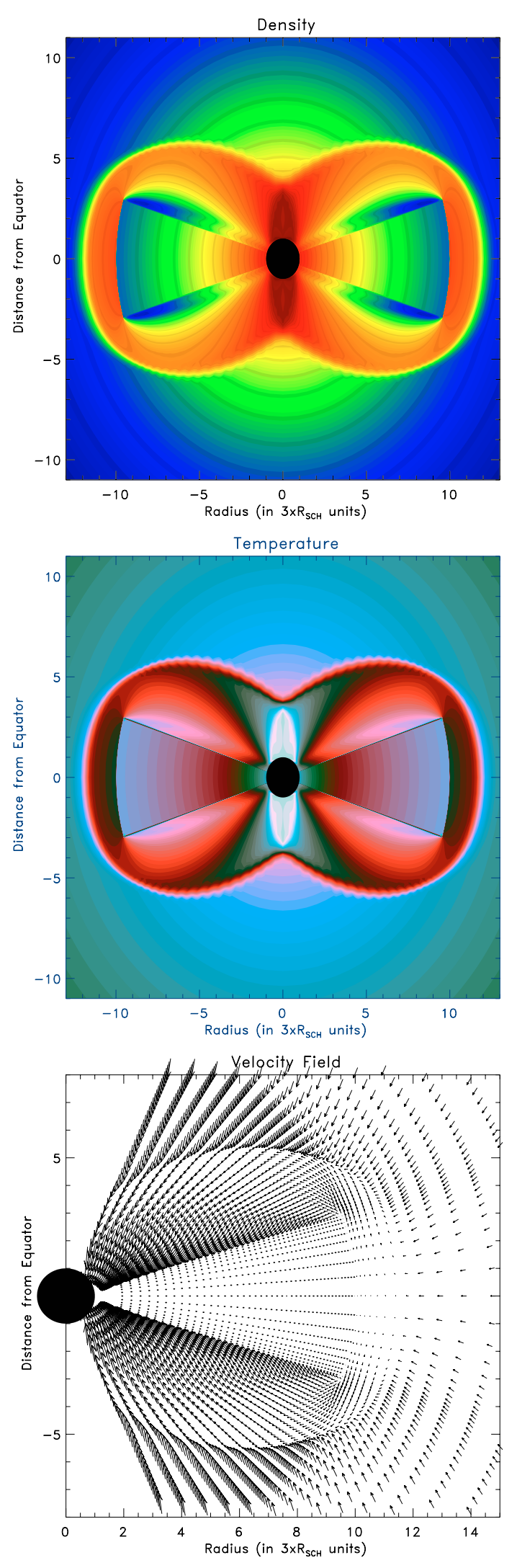

Fig. 8. Shock formation around a black hole surrounded by a cold disk. Top: the distribution of the velocity field. Middle: the density distribution (red: large values, blue: low values, green: intermediate values). Bottom: the temperature distribution (red: large values, blue: low values, gray: intermediate values). The curved shock front, where the temperature attains a maximum is obvious. 

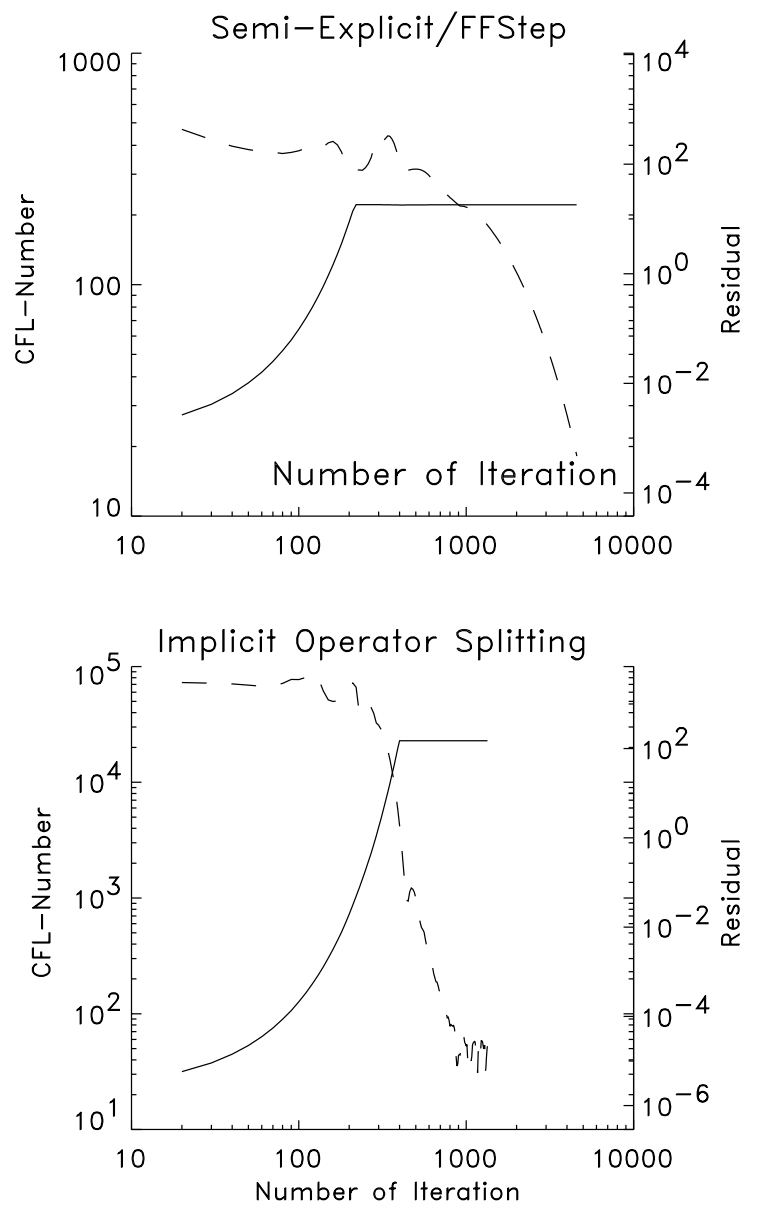

Fig. 9. Shock formation around a black hole surrounded by a cold disk. The flow problem is similar to the forward facing step (FFStep) in CFD. As in Fig. 3, the evolution of the CFL-number and the residual versus iteration number are shown. The semi-explicit method is apparently stable and converges to the stationary solution sought, even when large time steps that corresponds to a CFL-number of 220 are used. On the other hand, 1000 iterations were sufficient to obtain the same solution using the implicit operator-splitting approach (see Hujeirat \& Rannacher 2001).

The initial density distribution is taken to be a sine profile that moves to the right with velocity $U=1$ (see Fig. 10, plot a). As in plane geometry, the shape of the initial density profile is expected to be preserved as it moves right wards.

To compare results, a reference solution has been produced using an explicit solution procedure. The interval of calculation has been subdivided into 1000 finite volume cells, and a time step size that corresponds to $C F L=0.01$ has been used. The advection scheme here is third order accurate in space and second order in time.

Obviously, the semi-explicit method is capable of capturing the propagation of the wave front accurately (see Fig. 10). The amplitude of the wave is hardly changed, even when a time step size is used that corresponds to a CFL-number larger than unity. However, in the latter case several iterations per time step were required to assure convergence. For example, the profile "e" of Fig. 10 was obtained using $C F L=2.5$ in combination with two global iterations per time step. Nevertheless, this solution is clearly inaccurate. However, by increasing the iteration number from 2 to 7 a more accurate solutions has been obtained even for $C F L=5$ (see Fig. 10 /profile "g").

The profile "f" of Fig. 10 shows that even fully implicit methods may fail to reproduce the correct wave profile, if large CFL-numbers are used.

\section{The DuFort-Frankel and other schemes}

The Dufort-Frankel scheme is best suited for modelling diffusion-dominated flows and provides solutions of second order spatial and temporal accuracies (see Fletcher 1988 for further details). The scheme is unconditionally stable, as it relies on adding the positive coefficient resulting from finite difference discretization of the diffusion operator to the diagonal elements, thereby enhancing the diagonal dominance of the matrix coefficient.

Despite this similarity, the scheme still differs still from the semi-explicit method presented here at least in two aspects:

1. The DuFort-Frankel scheme is generically second order accurate in time. The scheme fails to be consistent when large time steps are used. Thus, the method is not suited for searching steady state solutions.

2. The scheme is not suited for modelling advectiondominated flows.

Another solution procedure that might look similar to the semiexplicit method presented here is the Jacobi iteration method. Inspection of Eq. (19), however, shows that neither the lower nor the upper diagonal matrices are considered in the present solution scheme. Furthermore, the consistency of our solution procedure with the original set of equations is guaranteed through adopting the defect-correction strategy. Unlike the Jacobi method, which may diverge if large time steps are used, the test calculations presented here show that the semi-explicit method converges even for relatively large CFL numbers.

\section{Summary}

In this paper we have presented a strategy for modifying explicit methods into unconditionally stable semi-explicit methods. This modification may have an impact on computational methods in astrophysics, as most of the solvers used here are explicit. Specifically, enhancing the robustness and enlarging the range of application of existing explicit methods can be made practically feasible. In particular, modifying explicit methods in this manner enable us to use them for searching strongly time-dependent as well as steady state solutions for the Euler or Navier-Stokes equations in multiple dimensions.

The new strategy is based on reformulating explicit methods in matrix form, i.e., transforming explicit methods from scalar into matrix-vector problems. Thus, solving the set of time-dependent HD or MHD equations would require solving the matrix equation $A q=b$. Explicit methods in this case rely on approximating and replacing the matrix $A$ by the most simple matrix in algebra: the identity matrix $I$. This solution procedure may converge if the time step size is sufficiently small, or equivalently, the entries of the matrix $I / \delta t$ are sufficiently 


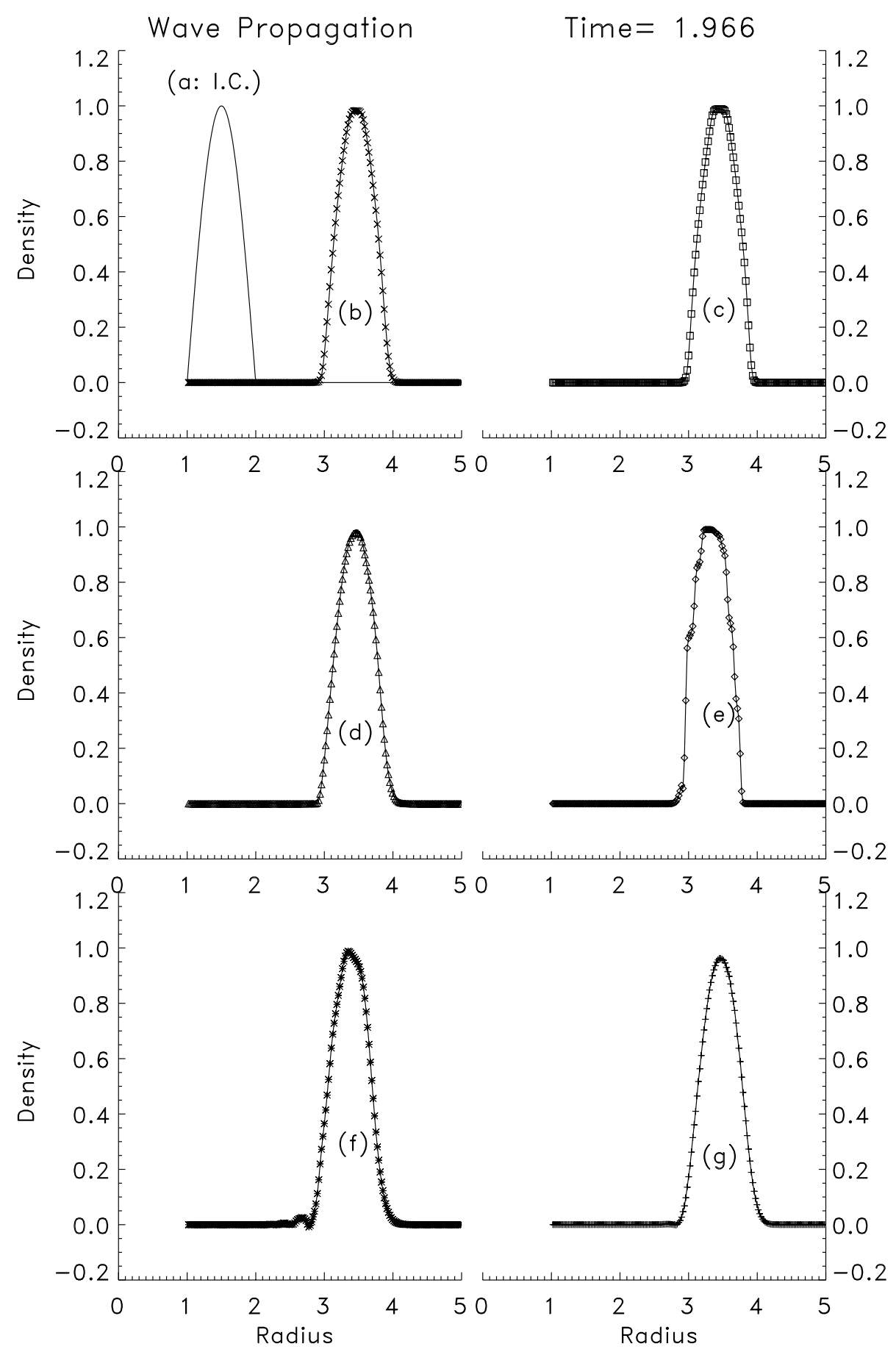

Fig. 10. The propagation of waves in one dimension. The initial density profile is the sine wave (a), which moves to the right with velocity $U=1$. The computed profiles "b" to "g" have been obtained after time $=1.966$, using 200 finite volume cells. The adopted advection scheme is of third order spatial accuracy and second order accuracy in time. The profiles "b, $\mathrm{d}, \mathrm{f}$ " have been obtained using the fully implicit solution procedure with time step sizes that correspond to $C F L=1,2.5$ and 5, respectively. The profiles "c, e, g" have been obtained using the semi-explicit approach with $C F L=1,2.5$ and 5, respectively.

large, so that all off-diagonal elements of $A$ can be safely neglected. As we have shown in Sect. 2 this is equivalent to the requirement that $C F L<1$.

The new modification, which led to the construction of the semi-explicit method, is based on replacing the abovementioned identity matrix by a diagonal matrix. From the results of the various test calculations presented here we conclude that the new method is consistent and numerically stable even when large CFL numbers are used. Moreover, the semi-explicit method presented here is capable of modelling viscous or inviscid flows governed by strong shocks.

Unlike normal explicit methods, in which inclusion of diffusion generally causes further limitations on the time step size, diffusion operators in the semi-explicit formulation enhance the 
diagonal dominance and the stability of the inversion procedure, irrespective of the dimensionality of the problem.

Additionally, we have shown that the residual smoothing approach accelerates the convergence of both explicit and semiexplicit methods.

It should be noted here that moving shocks are non-linear features of HD-flows, and in most cases the corresponding equations do not acquire stationary solutions. In this case, the time step size should be chosen sufficiently small to capture shock profiles accurately, irrespective of whether the method used is explicit or implicit. For modelling such flows, explicit methods are superior over implicit solvers, provided that the flow is isothermal/adiabatic or polytropic. If other physical and chemical processes are concerned, which generally operate on much shorter time scales than the dynamical time scale, implicit methods are much more appropriate.

\section{References}

Beam, R. M., \& Warming, R. F. 1978, AIAA, 16, 393

Enander, R. 1997, SIAM J. Sc. Comp., 18, 5, 1243

Fletcher, C. A. J. 1988, Computational Techniques for Flud Dynamics, vol. I, II (Springer-Verlag)

Hackbusch, W. 1994, Iterative Solution of Large Sparse Systems of Equations (New York-Berlin-Heidelberg: Springer-Verlag)

Hirsch, C. 1988, Numerical Computation of Internal and External Flows, vol. I, II (Joun Wiley \& Sons)

Hujeirat, A., \& Rannacher, R. 2001, New Astron. Rev., 45, 425

Koide, S., Shibata, K., \& Kudoh, T. 1999, ApJ, 522, 727

MacCormack, R. W. 1985, AIAA, Paper 85-0032

Richtmyer, R. D., \& Morton, K. W. 1967, Difference methods for initial value problems, 2 nd ed. (New York: Interscience)

Stone, J. M., \& Norman, M. L. 1992, ApJS, 80, 753

Swanson, R. C., \& Turkel, E. 1997, NASA, TP-3631, 81

Ziegler, U. 1998, Comp. Phys. Comm., 109, 111 